Supporting Information for:

\title{
LICAR: An application for isotopic correction of targeted lipidomic data acquired with class-based chromatographic separations using multiple reaction monitoring.
}

Liang Gao ${ }^{1,2}$, Shanshan $\mathrm{Ji}^{2}$, Bo Burla ${ }^{2}$, Markus R. Wenk ${ }^{1,2}$, Federico Torta ${ }^{1,2}$, and Amaury Cazenave-Gassiot ${ }^{1,2^{*}}$

1 Department of Biochemistry, Yong Loo Lin School of Medicine, National University of Singapore, Singapore

2 Singapore Lipidomics Incubator (SLING), Life Sciences Institute, National University of Singapore, Singapore.

* Address correspondence to: A.C.-G. at SLING, Life Sciences Institute, National University of Singapore, 28 Medical Drive \#02-15A, Singapore 117456. Tel. +6565168174; e-mail bchacgt@nus.edu.sg

\section{Contents}

Contents

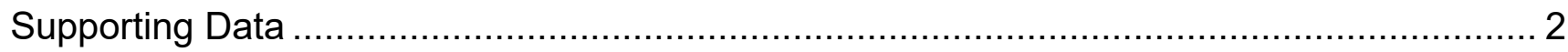

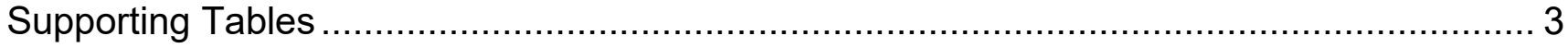

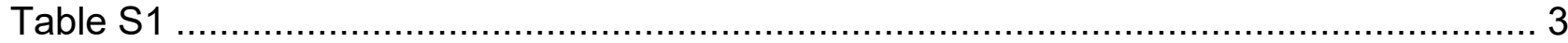

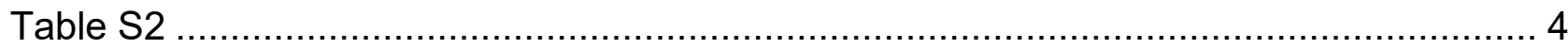

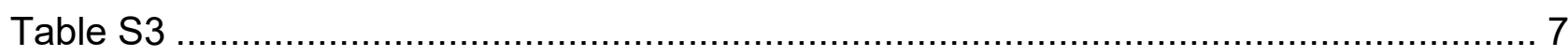

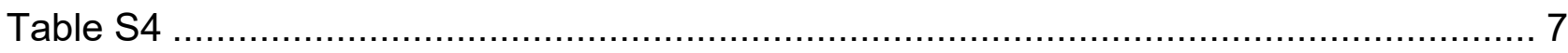

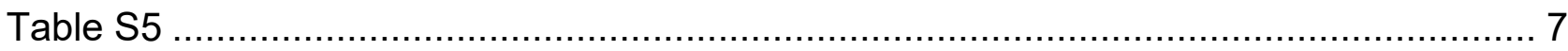

Supporting Figures

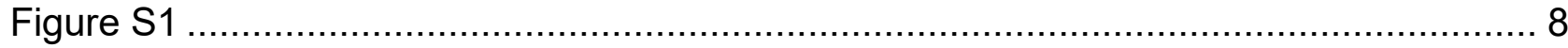

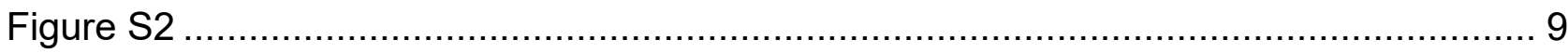

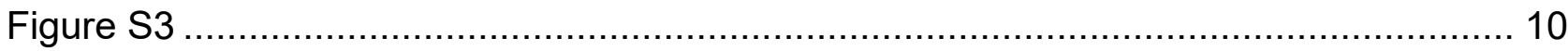

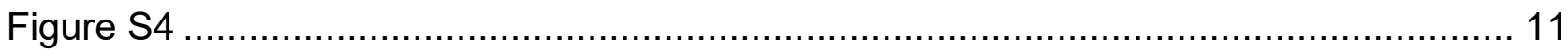

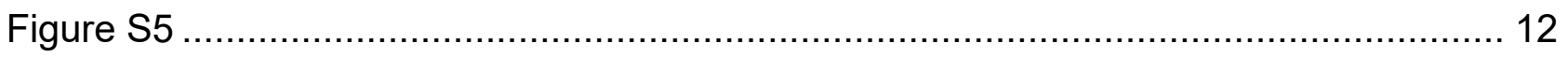




\section{Supporting Data}

LIDAR application, $\mathrm{R}$ scripts, and templates are available from https://github.com/SLINGhub/LICAR. The dataset used during the current study is available from the corresponding author.

Online Shiny app available at https://slinghub.shinyapps.io/LICAR/. 


\section{Supporting Tables}

\section{Table S1}

Formulae calculation for several lipid classes and MRM transitions types.

\begin{tabular}{|c|c|c|c|c|c|c|c|c|c|}
\hline \multirow{2}{*}{ Lipid class } & \multirow{2}{*}{ Abbreviation } & \multirow{2}{*}{ MRM pattern } & \multirow{2}{*}{ Condition } & \multirow{2}{*}{ Lipid } & \multicolumn{4}{|c|}{ Formula for calculation $\mathrm{C}_{\mathrm{x}} \mathrm{H}_{\mathrm{y}} \mathrm{O}_{\mathrm{z}} \mathrm{N}_{\mathrm{w}}$} & \multirow{2}{*}{ Overlap } \\
\hline & & & & & C & $\mathbf{H}$ & 0 & $\mathbf{N}$ & \\
\hline Lysophosphatidylcholine & LPC & $\begin{array}{l}{[\mathrm{M}+\mathrm{H}]^{+},} \\
\mathrm{HG} \text { product ion } \mathrm{m} / \mathrm{z} \\
184.1\end{array}$ & $\begin{array}{l}\text { Diff }_{\text {Pre }}=2 \\
\text { Diff }_{\text {Pro }}=0\end{array}$ & LPC 20:3 & $20+3$ & $C * 2-2-2 * 3$ & 3 & 0 & Neutral-fragment \\
\hline Lysoplasmanylcholine & LPCO & $\begin{array}{l}{[\mathrm{M}+\mathrm{H}]^{+},} \\
\mathrm{HG} \text { product ion } \mathrm{m} / \mathrm{z} \\
104.1\end{array}$ & $\begin{array}{l}\text { Differe }_{P r}=2 \\
\text { DiffPro }=0\end{array}$ & LPC O-20:4 & $20+3$ & $C * 2+1-2 * 4$ & 5 & 0 & Neutral-fragment \\
\hline Lysoplasmanylcholine & LPCOql & $\begin{array}{l}{[\mathrm{M}+\mathrm{H}]^{+}, \mathrm{HG} \text { qualifier }} \\
\text { product ion } \mathrm{m} / \mathrm{z} 184.1\end{array}$ & $\begin{array}{l}\text { Diff }_{\text {Pre }}=2 \\
\text { Diff }_{\text {Pro }}=0\end{array}$ & LPC 0-20:4 & $20+3$ & $C * 2-0-2 * 4$ & 2 & 0 & Neutral-fragment \\
\hline Phosphatidylcholine & $\mathrm{PC}$ & $\begin{array}{l}{[\mathrm{M}+\mathrm{H}]^{+},} \\
\mathrm{HG} \text { product ion } \mathrm{m} / \mathrm{z} \\
184.1\end{array}$ & $\begin{array}{l}\text { Diff }_{\text {Pre }}=2 \\
\text { Differo }=0\end{array}$ & PC 32:0 & $32+3$ & $C * 2-4-2 * 0$ & 4 & 0 & Neutral-fragment \\
\hline Sphingomyelin & SM & $\begin{array}{l}{[\mathrm{M}+\mathrm{H}]^{+},} \\
\mathrm{HG} \text { product ion } \mathrm{m} / \mathrm{z} \\
184.1\end{array}$ & $\begin{array}{l}\text { Differe }=2 \\
\text { DiffPro }=0\end{array}$ & SM 32:0 & 32 & $C * 2-1-2 * 0$ & 2 & 1 & Neutral-fragment \\
\hline Lysophosphatidylethanolamine & LPE & $\begin{array}{l}{[\mathrm{M}+\mathrm{H}]^{+},} \\
\mathrm{HG} N L \text { of } m / z 141\end{array}$ & $\begin{array}{l}\text { Differe }=2 \\
\text { Differo }=2\end{array}$ & LPE 20:3 & $20+3$ & $C * 2-1-2 * 3$ & 3 & 0 & Product-ion \\
\hline Phosphatidylethanolamine & PE & $\begin{array}{l}{[\mathrm{M}+\mathrm{H}]^{+}} \\
\mathrm{HG} \mathrm{NL} \text { of } \mathrm{m} / \mathrm{z} 141\end{array}$ & $\begin{array}{l}\text { Diff }_{\text {Pre }}=2 \\
\text { DiffPro }=2\end{array}$ & PE 32:0 & $32+3$ & $C * 2-3-2 * 0$ & 4 & 0 & Product-ion \\
\hline Phosphatidylinositol & $\mathrm{PI}$ & $\begin{array}{l}{\left[\mathrm{M}+\mathrm{NH}_{4}\right]^{+},} \\
\mathrm{HG} \mathrm{NL} \text { of } m / z 277\end{array}$ & $\begin{array}{l}\text { Differe }=2 \\
\text { DiffPro }=2\end{array}$ & PI 32:0 & $32+3$ & $C * 2-3-2 * 0$ & 4 & 0 & Product-ion \\
\hline Phosphatidylglycerol & PG & $\begin{array}{l}{\left[\mathrm{M}+\mathrm{NH}_{4}\right]^{+},} \\
\mathrm{HG} \mathrm{NL} \text { of } m / z 189\end{array}$ & $\begin{array}{l}\text { Diff }_{\text {Pre }}=2 \\
\text { Diff }_{\text {Pro }}=2\end{array}$ & PG 32:0 & $32+3$ & $C * 2-3-2 * 0$ & 4 & 0 & Product-ion \\
\hline Phosphatidylserine & PS & $\begin{array}{l}{\left[\mathrm{M}+\mathrm{NH}_{4}\right]^{+},} \\
\mathrm{HG} \mathrm{NL} \text { of } m / z 185\end{array}$ & $\begin{array}{l}\text { DiffPre }=2 \\
\text { DiffPro }=2\end{array}$ & PS 32:0 & $32+3$ & $C * 2-3-2{ }^{*} 0$ & 4 & 0 & Product-ion \\
\hline Lysophosphatidylethanolamine & LPENHG & $\begin{array}{l}{[\mathrm{M}-\mathrm{H}]} \\
\text { HG product ion } m / z \\
196.1\end{array}$ & $\begin{array}{l}\text { DiffPre }=2 \\
\text { Differo }=0\end{array}$ & LPE 18:2 & 18 & $C * 2-0-2 * 2$ & 2 & 0 & Neutral-fragment \\
\hline Phosphatidylethanolamine & PENHG & $\begin{array}{l}{[\mathrm{M}-\mathrm{H}]^{-} \text {, }} \\
\mathrm{HG} \text { product ion } \mathrm{m} / \mathrm{z} \\
196.1\end{array}$ & $\begin{array}{l}\text { Diff }_{\text {Pre }}=2 \\
\text { Diff }_{\text {Pro }}=0\end{array}$ & PE 36:2 & 36 & $C * 2-2-2 * 2$ & 3 & 0 & Neutral-fragment \\
\hline Phosphatidylinositol & PINHG & $\begin{array}{l}{[\mathrm{M}-\mathrm{H}]^{-}} \\
\text {HG product ion } m / z 241\end{array}$ & $\begin{array}{l}\text { Differe }=2 \\
\text { DiffPro }=0\end{array}$ & $\mathrm{PI} 34: 2$ & $34+3$ & $C * 2-2-2 * 2$ & 5 & 0 & Neutral-fragment \\
\hline Phosphatidylglycerol & PGNHG & $\begin{array}{l}{[\mathrm{M}-\mathrm{H}]^{-},} \\
\text {HG product ion } \mathrm{m} / \mathrm{z} 153\end{array}$ & $\begin{array}{l}\text { Differe }=2 \\
\text { Diff }_{\text {Pro }}=0\end{array}$ & PG 34:2 & $34+3$ & $C * 2-2-2 * 2$ & 5 & 0 & Neutral-fragment \\
\hline Phosphatidylserine & PSNHG & $\begin{array}{l}{[\mathrm{M}-\mathrm{H}]^{-}} \\
\mathrm{HG} \text { NL of } m / z 87\end{array}$ & $\begin{array}{l}\text { Diff }_{\text {Pre }}=2 \\
\text { Diff }_{\text {Pro }}=2\end{array}$ & PS 36:2 & $36+3$ & $C * 2-2-2 * 2$ & 8 & 0 & Product-ion \\
\hline \multirow{2}{*}{ Plasmenylethanolamine } & \multirow{2}{*}{ PEP } & \multirow{2}{*}{$\begin{array}{l}{[\mathrm{M}+\mathrm{H}]^{+}, \text {FA product ion }} \\
{\left[\mathrm{FA}+\mathrm{C}_{3} \mathrm{H}_{4} \mathrm{O}+\mathrm{H}\right]^{+}}\end{array}$} & $\begin{array}{l}\text { DiffPre }=2, \\
\text { DiffPro }=0\end{array}$ & PE P-20:0/20:4 & $20+2$ & $C * 2+2-2 * 0$ & 4 & 1 & Neutral-fragment \\
\hline & & & $\begin{array}{l}\text { Diff }_{\text {Pre }}=2 \\
\text { Diff }_{\text {Pro }}=2\end{array}$ & PE P-20:0/20:4 & $20+3$ & $C * 2-1-2 * 4$ & 3 & 0 & Product-ion \\
\hline \multirow{2}{*}{ Phosphatidylcholine } & \multirow{2}{*}{ PCNFA } & \multirow{2}{*}{$\begin{array}{l}{\left[\mathrm{M}+\mathrm{CHOO}^{-}\right.} \\
\text {FA product ion }[\mathrm{FA}-\mathrm{H}]^{-}\end{array}$} & $\begin{array}{l}\text { Diff }_{\text {Pre }}=2 \\
\text { Diff }\end{array}$ & PC 18:1/16:1 & $18+9$ & $C * 2-0-2 * 1$ & 8 & 1 & Neutral-fragment \\
\hline & & & $\begin{array}{l}\text { DiffPre }=2 \\
\text { DiffPro }=2\end{array}$ & PC 16:0/18:2 & 18 & $C * 2-1-2 * 2$ & 2 & 0 & Product-ion \\
\hline \multirow{2}{*}{ Phosphatidylethanolamine } & \multirow{2}{*}{ PENFA } & \multirow{2}{*}{$\begin{array}{l}{[\mathrm{M}-\mathrm{H}]^{-}} \\
\text {FA product ion }[\mathrm{FA}-\mathrm{H}]^{-}\end{array}$} & $\begin{array}{l}\text { Diff } \text { pre }=2, \\
\text { Differo }=0\end{array}$ & PE 18:1/16:1 & $18+5$ & $C * 2-0-2 * 1$ & 6 & 1 & Neutral-fragment \\
\hline & & & $\begin{array}{l}\text { Diff }_{\text {Pre }}=2 \\
\text { Diff }_{\text {Pro }}=2\end{array}$ & PE 16:0/18:2 & 18 & $C * 2-1-2 * 2$ & 2 & 0 & Product-ion \\
\hline \multirow{2}{*}{ Phosphatidylglycerol } & \multirow{2}{*}{ PGNFA } & \multirow{2}{*}{$\begin{array}{l}{[\mathrm{M}-\mathrm{H}]^{-}} \\
\text {FA product ion }[\mathrm{FA}-\mathrm{H}]^{-}\end{array}$} & $\begin{array}{l}\text { Differe }=2 \\
\text { DiffPro }=0\end{array}$ & PG 18:1/16:1 & $18+6$ & $C * 2-1-2 * 1$ & 8 & 0 & Neutral-fragment \\
\hline & & & $\begin{array}{l}\text { Diff }_{\text {Pre }}=2 \\
\text { Diff }_{\text {Pro }}=2\end{array}$ & PG 16:0/18:2 & 18 & $C * 2-1-2 * 2$ & 2 & 0 & Product-ion \\
\hline \multirow{2}{*}{ Phosphatidylinositol } & \multirow{2}{*}{ PINFA } & \multirow{2}{*}{$\begin{array}{l}{[\mathrm{M}-\mathrm{H}]^{-}} \\
\text {FA product ion }[\mathrm{FA}-\mathrm{H}]^{-}\end{array}$} & 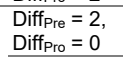 & PI 18:1/16:1 & $18+9$ & $C * 2-3-2 * 1$ & 11 & 0 & Neutral-fragment \\
\hline & & & $\begin{array}{l}\text { DiffPre }=2 \\
\text { DiffPro }=2\end{array}$ & PI 16:0/18:2 & 18 & $C * 2-1-2 * 2$ & 2 & 0 & Product-ion \\
\hline \multirow{2}{*}{ Phosphatidylserine } & \multirow{2}{*}{ PSNFA } & \multirow{2}{*}{$\begin{array}{l}{[\mathrm{M}-\mathrm{H}]^{-}} \\
\text {FA product ion }[\mathrm{FA}-\mathrm{H}]^{-}\end{array}$} & $\begin{array}{l}\text { Diff } \text { pre }=2, \\
\text { Differo }=0\end{array}$ & PS 18:1/16:1 & $18+6$ & $C * 2-2-2 * 1$ & 8 & 1 & Neutral-fragment \\
\hline & & & $\begin{array}{l}\text { Diff }_{\text {Pre }}=2 \\
\text { Diff }_{\text {Pro }}=2\end{array}$ & PS 16:0/18:2 & 18 & $C * 2-1-2 * 2$ & 2 & 0 & Product-ion \\
\hline \multirow{2}{*}{ Dehydroxyceramide } & & {$[\mathrm{M}+\mathrm{H}]^{+}$, } & $\begin{array}{l}\text { Differe }=2 \\
\text { Differo }=0\end{array}$ & Cer d18:0/16:1 & 16 & $C * 2+0-2 * 1$ & 2 & 0 & Neutral-fragment \\
\hline & dhCer & $\begin{array}{l}\text { LCB product ion } \\
{\left[\text { LCB- } \mathrm{H}_{2} \mathrm{O}+\mathrm{H}\right]^{+}}\end{array}$ & $\begin{array}{l}\text { Diff }_{\text {Pre }}=2 \\
\text { Diff }_{\text {Pro }}=2\end{array}$ & Cer d18:0/16:1 & 18 & $C * 2+2-2 * 1$ & 1 & 1 & Product-ion \\
\hline & Cer & {$[\mathrm{M}+\mathrm{H}]^{+}$, } & 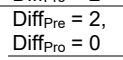 & Cer d18:1/16:1 & 16 & $C * 2+2-2 * 1$ & 3 & 0 & Neutral-fragment \\
\hline Ceramide & Cer & $\begin{array}{l}\text { LCB product Ion } \\
\left.\text { LCB- } 2 \mathrm{H}_{2} \mathrm{O}+\mathrm{H}\right]^{+}\end{array}$ & $\begin{array}{l}\text { Diff }_{\text {Pre }}=2, \\
\text { Diff Pro }=2\end{array}$ & Cer d18:1/16:1 & 18 & $C * 2+0-2 * 1$ & 0 & 1 & Product-ion \\
\hline & & {$[\mathrm{M}+\mathrm{H}]^{+}$, } & 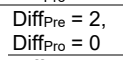 & $\begin{array}{c}\text { Hex1Cer } \\
\text { d18:1/16:1 }\end{array}$ & $16+6$ & $C * 2+0-2 * 1$ & 8 & 0 & Neutral-fragment \\
\hline Monohexosylceramide & Hex1Cer & $\begin{array}{l}\text { LCB product ion } \\
\left.\text { [LCB- } 2 \mathrm{H}_{2} \mathrm{O}+\mathrm{H}\right]^{+}\end{array}$ & $\begin{array}{l}\text { DiffPre }=2, \\
\text { DiffPro }=2\end{array}$ & $\begin{array}{c}\text { Hex1Cer } \\
\text { d18:1/16:1 }\end{array}$ & 18 & $C * 2+0-2 * 1$ & 0 & 1 & Product-ion \\
\hline & & {$[\mathrm{M}+\mathrm{H}]^{+}$, } & $\begin{array}{l}\text { Diff Pre }=2, \\
\text { Differo }=0\end{array}$ & $\begin{array}{c}\text { Hex2Cer } \\
\text { d18:1/16:1 }\end{array}$ & $16+12$ & $C * 2-2-2 * 1$ & 13 & 0 & Neutral-fragment \\
\hline Dihexosylceramide & Hex2Cer & 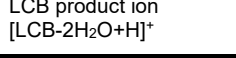 & $\begin{array}{l}\text { Diff }_{\text {Pre }}=2, \\
\text { DiffPro }=2\end{array}$ & $\begin{array}{c}\text { Hex2Cer } \\
\text { d18:1/16:1 }\end{array}$ & 18 & $C * 2+0-2 * 1$ & 0 & 1 & Product-ion \\
\hline
\end{tabular}

Notes: $\mathrm{HG}=$ headgroup, $\mathrm{FA}=$ fatty acyl, $\mathrm{LCB}=$ long chain base, $\mathrm{NL}=$ neutral loss. 


\section{Table S2}

Theoretical and experimental isotopic contributions for commonly used internal standards.

\begin{tabular}{|c|c|c|c|c|c|c|c|c|c|c|c|c|c|}
\hline \multirow[b]{2}{*}{ Lipid name } & \multirow[b]{2}{*}{ Polarity } & \multirow[b]{2}{*}{$\begin{array}{l}\text { Precursor } \\
\text { ion } m / z\end{array}$} & \multirow[b]{2}{*}{$\begin{array}{l}\text { Product } \\
\text { ion } m / z\end{array}$} & \multicolumn{2}{|c|}{ Precursor-level correction } & \multicolumn{4}{|c|}{ Neutral-fragment overlap $\left(\right.$ Diff $\left._{\mathrm{Pro}}=0\right)$} & \multicolumn{4}{|c|}{ Product-ion overlap $\left(\right.$ Diff $\left._{\mathrm{Pro}}=2\right)$} \\
\hline & & & & $\begin{array}{l}\text { Precursor's } \\
\text { formula }\end{array}$ & $\begin{array}{c}\text { Calculated } \\
\text { isotopic } \\
\text { contribution }(\%)^{1}\end{array}$ & $\begin{array}{l}\text { Affected } \\
\text { transition }\end{array}$ & $\begin{array}{l}\text { Relevant neutral } \\
\text { fragment's } \\
\text { formula }\end{array}$ & $\begin{array}{l}\text { Calculated isotopic } \\
\text { contribution }(\%)^{2}\end{array}$ & $\begin{array}{c}\text { Experimental } \\
\text { isotopic } \\
\text { contribution }(\%)^{3}\end{array}$ & $\begin{array}{l}\text { Affected } \\
\text { transition }\end{array}$ & $\begin{array}{c}\text { Relevant } \\
\text { product's formula }\end{array}$ & $\begin{array}{c}\text { Calculated } \\
\text { isotopic } \\
\text { contribution (\%) }\end{array}$ & $\begin{array}{l}\text { Experimental isotopic } \\
\text { contribution }(\%)^{3}\end{array}$ \\
\hline LPC 20:0 & Positive & 552.4 & 184.1 & $\mathrm{C}_{28} \mathrm{H}_{59} \mathrm{NO}_{7} \mathrm{P}$ & 6.28 & 554.4 -> 184.1 & $\mathrm{C}_{23} \mathrm{H}_{44} \mathrm{O}_{3}$ & 3.73 & $3.69 \pm 0.79$ & & & & \\
\hline PC 28:0 & Positive & 678.5 & 184.1 & $\mathrm{C}_{36} \mathrm{H}_{73} \mathrm{NO}_{8} \mathrm{P}$ & 9.63 & 680.5 -> 184.1 & $\mathrm{C}_{31} \mathrm{H}_{58} \mathrm{O}_{4}$ & 6.54 & $6.65 \pm 0.73$ & & & & \\
\hline SM 30:1 & Positive & 647.5 & 184.1 & $\mathrm{C}_{35} \mathrm{H}_{72} \mathrm{~N}_{2} \mathrm{O}_{6} \mathrm{P}$ & 8.90 & 649.5 -> 184.1 & $\mathrm{C}_{30} \mathrm{H}_{57} \mathrm{O}_{2} \mathrm{~N}_{1}$ & 5.86 & $5.61 \pm 0.34$ & & & & \\
\hline LPE 14:0 & Positive & 426.3 & 285.3 & $\mathrm{C}_{19} \mathrm{H}_{41} \mathrm{NO}_{7} \mathrm{P}$ & 3.68 & & & & & $428.3->287.3$ & $\mathrm{C}_{17} \mathrm{H}_{33} \mathrm{O}_{3}$ & 2.30 & $2.43 \pm 0.18$ \\
\hline PE 28:0 & Positive & 636.5 & 495.5 & $\mathrm{C}_{33} \mathrm{H}_{67} \mathrm{NO}_{8} \mathrm{P}$ & 8.36 & & & & & $638.5->497.5$ & $\mathrm{C}_{31} \mathrm{H}_{59} \mathrm{O}_{4}$ & 6.54 & $6.64 \pm 0.96$ \\
\hline PG 28:0 & Positive & 684.5 & 495.5 & $\mathrm{C}_{34} \mathrm{H}_{71} \mathrm{NO}_{10} \mathrm{P}$ & 9.22 & & & & & $686.5->497.5$ & $\mathrm{C}_{31} \mathrm{H}_{59} \mathrm{O}_{4}$ & 6.54 & $6.41 \pm 0.48$ \\
\hline PI 25:0 & Positive & 730.4 & 453.4 & $\mathrm{C}_{34} \mathrm{H}_{69} \mathrm{NO}_{13} \mathrm{P}$ & 9.86 & & & & & $732.4->455.4$ & $\mathrm{C}_{28} \mathrm{H}_{53} \mathrm{O}_{4}$ & 5.48 & $5.20 \pm 0.18$ \\
\hline PS 28:0 & Positive & 680.5 & 495.5 & $\mathrm{C}_{34} \mathrm{H}_{67} \mathrm{NO}_{10} \mathrm{P}$ & 9.20 & & & & & 682.5 -> 497.5 & $\mathrm{C}_{31} \mathrm{H}_{59} \mathrm{O}_{4}$ & 6.54 & $6.61 \pm 0.53$ \\
\hline PE P-18:0/20:4 & Positive & 752.5 & 361.3 & $\mathrm{C}_{43} \mathrm{H}_{79} \mathrm{NO}_{7} \mathrm{P}$ & 12.75 & 754.5 -> 361.3 & $\mathrm{C}_{20} \mathrm{H}_{42} \mathrm{O}_{4} \mathrm{~N}_{1} \mathrm{P}_{1}$ & 3.27 & $3.04 \pm 0.12$ & 754.5 -> 363.3 & $\mathrm{C}_{23} \mathrm{H}_{37} \mathrm{O}_{3}$ & 3.71 & $3.81 \pm 0.12$ \\
\hline Cer d18:0/8:0 & Positive & 428.4 & 284.3 & $\mathrm{C}_{26} \mathrm{H}_{54} \mathrm{NO}_{3}$ & 4.75 & 430.4 -> 284.3 & $\mathrm{C}_{8} \mathrm{H}_{16} \mathrm{O}_{2}$ & 0.76 & $0.79 \pm 0.44$ & $430.4->286.3$ & $\mathrm{C}_{18} \mathrm{H}_{38} \mathrm{O}_{1} \mathrm{~N}_{1}$ & 2.16 & $2.23 \pm 0.92$ \\
\hline Cer d18:1/17:0 & Positive & 552.5 & 264.3 & $\mathrm{C}_{35} \mathrm{H}_{7} \mathrm{NO}_{3}$ & 8.09 & 554.5 -> 264.3 & $\mathrm{C}_{17} \mathrm{H}_{36} \mathrm{O}_{3}$ & 2.37 & $2.23 \pm 0.12$ & 554.5 -> 266.3 & $\mathrm{C}_{18} \mathrm{H}_{34} \mathrm{~N}_{1}$ & 1.94 & $1.77 \pm 0.08$ \\
\hline $\begin{array}{l}\text { Hex1Cer } \\
\text { d18:1/8:0 }\end{array}$ & Positive & 588.8 & 264.3 & $\mathrm{C}_{32} \mathrm{H}_{62} \mathrm{NO}_{8}$ & 7.95 & 590.8 -> 264.3 & $\mathrm{C}_{14} \mathrm{H}_{28} \mathrm{O}_{8}$ & 2.81 & $2.56 \pm 0.17$ & 590.8 -> 266.3 & $\mathrm{C}_{18} \mathrm{H}_{34} \mathrm{~N}_{1}$ & 1.94 & $2.00 \pm 0.23$ \\
\hline $\begin{array}{l}\text { Fex<Cer } \\
\text { d18:1/8:0 }\end{array}$ & Positive & 750.6 & 264.3 & $\mathrm{C}_{38} \mathrm{H}_{72} \mathrm{NO}_{13}$ & 11.62 & 752.6 -> 264.3 & $\mathrm{C}_{20} \mathrm{H}_{38} \mathrm{O}_{13}$ & 5.10 & $4.76 \pm 0.11$ & 752.6 -> 266.3 & $\mathrm{C}_{18} \mathrm{H}_{34} \mathrm{~N}_{1}$ & 1.94 & $1.87 \pm 0.10$ \\
\hline LPE 14:0 & Negative & 424.3 & 196 & $\mathrm{C}_{19} \mathrm{H}_{39} \mathrm{NO}_{7} \mathrm{P}$ & 3.68 & $426.3->196$ & $\mathrm{C}_{14} \mathrm{H}_{28} \mathrm{O}_{2}$ & 1.54 & $1.82 \pm 0.28$ & & & & \\
\hline PE 28:0 & Negative & 634.5 & 196 & $\mathrm{C}_{33} \mathrm{H}_{65} \mathrm{NO}_{8} \mathrm{P}$ & 8.35 & $636.5->196$ & $\mathrm{C}_{28} \mathrm{H}_{54} \mathrm{O}_{3}$ & 5.26 & $5.68 \pm 1.67$ & & & & \\
\hline PG 28:0 & Negative & 665.5 & 153 & $\mathrm{C}_{34} \mathrm{H}_{66} \mathrm{O}_{10} \mathrm{P}$ & 9.06 & $667.5->153$ & $\mathrm{C}_{31} \mathrm{H}_{60} \mathrm{O}_{5}$ & 6.77 & $6.20 \pm 0.14$ & & & & \\
\hline PI 25:0 & Negative & 711.5 & 241 & $\mathrm{C}_{34} \mathrm{H}_{64} \mathrm{O}_{13} \mathrm{P}$ & 9.71 & $713.5->241$ & $\mathrm{C}_{28} \mathrm{H}_{54} \mathrm{O}_{5}$ & 5.70 & $5.80 \pm 0.41$ & & & & \\
\hline PS 28:0 & Negative & 678.5 & 591.5 & $\mathrm{C}_{34} \mathrm{H}_{65} \mathrm{NO}_{10} \mathrm{P}$ & 9.19 & & & & & $680.5>593.5$ & $\mathrm{C}_{31} \mathrm{H}_{60} \mathrm{O}_{8} \mathrm{P}_{1}$ & 7.42 & $7.51 \pm 0.83$ \\
\hline PC 14:0/14:0 & Negative & 722.5 & 227.2 & $\mathrm{C}_{37} \mathrm{H}_{73} \mathrm{NO}_{10} \mathrm{P}$ & 10.51 & 724.5 -> 227.2 & $\mathrm{C}_{23} \mathrm{H}_{46} \mathrm{O}_{8} \mathrm{~N}_{1} \mathrm{P}_{1}$ & 4.91 & $4.34 \pm 0.69$ & 724.5 -> 229.2 & $\mathrm{C}_{14} \mathrm{H}_{27} \mathrm{O}_{2}$ & 1.53 & $1.80 \pm 0.63$ \\
\hline PE 14:0/14:0 & Negative & 634.5 & 227.2 & $\mathrm{C}_{33} \mathrm{H}_{65} \mathrm{NO}_{8} \mathrm{P}$ & 8.35 & 636.5 -> 227.2 & $\mathrm{C}_{19} \mathrm{H}_{38} \mathrm{O}_{6} \mathrm{~N}_{1} \mathrm{P}_{1}$ & 3.45 & $3.15 \pm 0.25$ & 636.5 -> 229.2 & $\mathrm{C}_{14} \mathrm{H}_{27} \mathrm{O}_{2}$ & 1.53 & $1.65 \pm 0.28$ \\
\hline PG 14:0/14:0 & Negative & 665.5 & 227.2 & $\mathrm{C}_{34} \mathrm{H}_{66} \mathrm{O}_{10} \mathrm{P}$ & 9.06 & 667.5 -> 227.2 & $\mathrm{C}_{20} \mathrm{H}_{3} 9 \mathrm{O}_{8} \mathrm{P}_{1}$ & 4.03 & $3.64 \pm 0.13$ & 667.5 -> 229.2 & $\mathrm{C}_{14} \mathrm{H}_{27} \mathrm{O}_{2}$ & 1.53 & $1.54 \pm 0.13$ \\
\hline PI 13:0/12:0 & Negative & 711.5 & 199.2 & $\mathrm{C}_{34} \mathrm{H}_{64} \mathrm{O}_{13} \mathrm{P}$ & 9.71 & 713.5 -> 199.2 & $\mathrm{C}_{22} \mathrm{H}_{41} \mathrm{O}_{11} \mathrm{P}_{1}$ & 5.18 & $5.28 \pm 0.64$ & 713.5 -> 201.2 & $\mathrm{C}_{12} \mathrm{H}_{23} \mathrm{O}_{2}$ & 1.23 & $1.33 \pm 0.31$ \\
\hline PS 14:0/14:0 & Negative & 678.5 & 227.2 & $\mathrm{C}_{34} \mathrm{H}_{65} \mathrm{NO}_{10} \mathrm{P}$ & 9.19 & 680.5 -> 227.2 & $\mathrm{C}_{20} \mathrm{H}_{38} \mathrm{O}_{8} \mathrm{~N}_{1} \mathrm{P}_{1}$ & 4.11 & $4.14 \pm 0.44$ & 680.5 -> 229.2 & $\mathrm{C}_{14} \mathrm{H}_{27} \mathrm{O}_{2}$ & 1.53 & $1.53 \pm 0.44$ \\
\hline
\end{tabular}

${ }_{2}^{1}$ Calculated with enviPat based on precursor's formula

${ }^{3}$ Measured AUC of the affected transition as a percentage of the interfering transition 


\section{Table S3}

MRM lists.

Provided as a Microsoft Excel file.

\section{Table S4}

Raw data.

Provided as a Microsoft Excel file.

\section{Table S5}

Examples of templates to create .csv files.

Provided as a Microsoft Excel file. 
Supporting Figures

\section{Figure $\$ 1$}

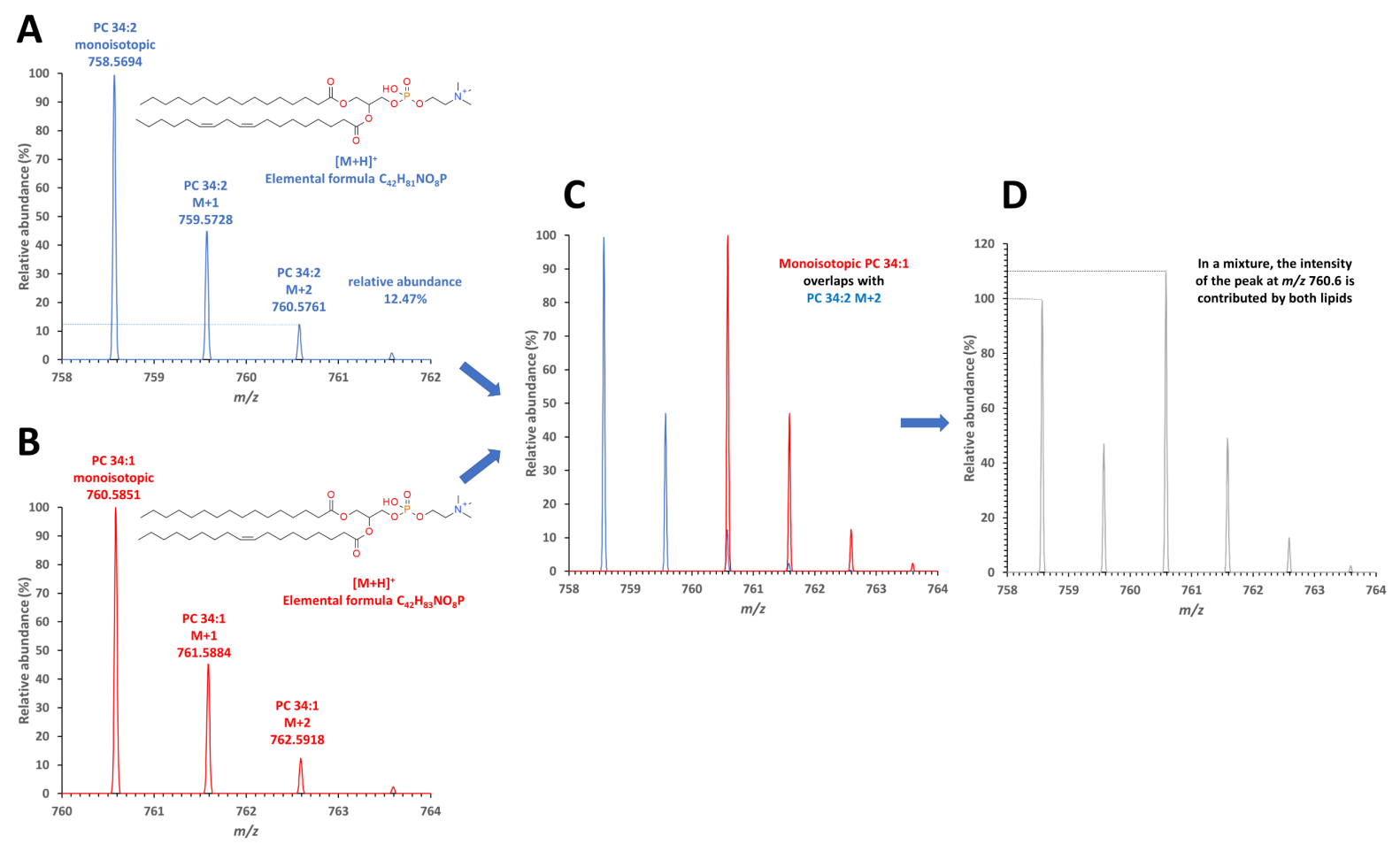

Figure S1. Example of isotopic overlap in single stage mass spectrum. A) Mass spectrum of PC 34:2 obtained in a QToF instrument; B) Mass spectrum of PC 34:1 obtained in a QToF instrument; C) Overlay of spectra in A and B; and D) Simulated mass spectrum illustrating how the intensity of the peak at $\mathrm{m} / \mathrm{z} 762.6$ is influence by isotopic overlap. 


\section{Figure S2}

\section{A}

PE 36:2 [M+H] ${ }^{+}$ MRM transition: 744.6 -> 603.6<smiles>CCCCC/C=C\C/C=C\CCCCCCCCC(=O)OC(COC(=O)CCCCCCCCCCCCCCCCC)COP(=O)(O)OCC[NH3+]</smiles>

PE 36:1 [M+H] $]^{+}$ MRM transition: 746.6 -> 605.6<smiles>CCCCCCCCCCCCCCCCCC(=O)OCC(COP(=O)(O)OCC[NH3+])C(CC)OC(=O)CCCCCCCCCCCCCC</smiles>

Charged fragment $m / z$ precursor-141.0
Neutral ethanolamine fragment

B

\begin{tabular}{|c|c|c|c|c|c|}
\hline & $\begin{array}{l}\text { Number of }{ }^{13} \mathrm{C} \text { in } \\
\text { charged fragment }\end{array}$ & $\begin{array}{l}\text { Number of }{ }^{13} \mathrm{C} \text { in head- } \\
\text { group fragment }{ }^{+}\end{array}$ & Precursor $m / z$ & Fragment $m / z$ & $\begin{array}{c}\text { Interference with PE 36:1 } \\
746->605.6 \text { transition }\end{array}$ \\
\hline \multirow{4}{*}{ PE 36:2 } & 0 & 0 & 744 & 603 & N/A \\
\hline & 1 & 1 & 745 & 604 & NO \\
\hline & 0 & 2 & 746 & 603 & NO \\
\hline & 2 & 0 & 746 & 605 & YES \\
\hline
\end{tabular}

${ }^{+13} \mathrm{C}$ is used as an example here for simplicity's sake and because it is the most significant contributor to isotopic overlap, but other naturally occurring heavy isotopes are considered in all calculations

Figure S2. Isotopic overlap in MRM transitions using headgroup. A) Structures of PE 36:2 and PE 36:1 showing MS/MS fragments; B) Table illustrating in which case(s) isotopic overlap occurs in MRM analysis. 


\section{Figure S3}

A

PC 16:1_18:1 [M+HCOO]

MRM transitions:

$802.6->253.2$

$802.6->281.2$

PC 16:0_18:2 [M+HCOO]

MRM transitions:

$802.6->255.2$

$802.6->279.2$

PC 16:0_18:1 [M+HCOO]

MRM transitions:

$804.6->255.2$

804.6 -> 281.2

PC 16:1_18:0 [M+HCOO] MRM transitions:

804.6 -> 253.2

804.6 -> 283.2

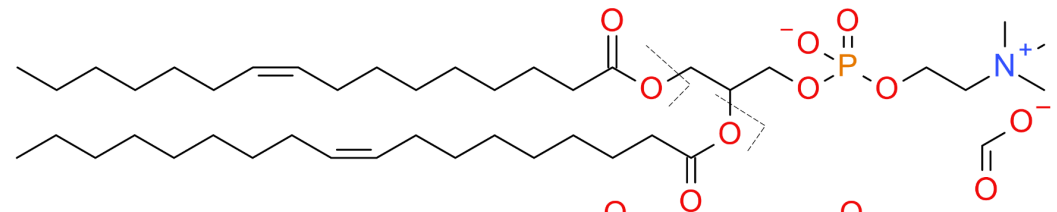<smiles>CCCCC=CCC=CCCCCCCCC(=O)OC(C)C(COC(=O)CCCCCCCCCCCCCCC)COP(=O)(OC)OCC[N+](C)(C)C</smiles><smiles>[CH2]OP(=O)(OCC[N+](C)(C)O)OCC(COC(=O)CCCCCCCC/C=C\CCCCCCCCC)OC(=O)CCCCCCCCCCCCC</smiles><smiles>CCCCCCCCCCCCCCCC(=O)OCC(COP(=O)(OC)OCC[N+](C)(C)C)C(C)(COC(=O)CCCCCCCCCC)OC</smiles>

B

\begin{tabular}{|c|c|c|c|c|c|c|}
\hline & $\begin{array}{l}\text { Number of }{ }^{13} \mathrm{C} \text { in } \\
\text { sn } 1 \mathrm{FA}^{+}\end{array}$ & $\begin{array}{l}\text { Number of }{ }^{13} \mathrm{C} \text { in } \\
\text { sn } 2 \mathrm{FA}^{+}\end{array}$ & $\begin{array}{l}\text { Number of }{ }^{13} \mathrm{C} \text { in glycerol- } \\
\text { headgroup }\end{array}$ & $\begin{array}{l}\text { Precursor } \\
m / z\end{array}$ & FA fragments $m / z$ & Interferences with other species \\
\hline \multirow{14}{*}{ PC 16:1_18:1 } & \multirow{2}{*}{0} & \multirow{2}{*}{0} & \multirow{2}{*}{0} & \multirow{2}{*}{802} & 253 & N/A \\
\hline & & & & & 281 & N/A \\
\hline & \multirow{2}{*}{1} & \multirow{2}{*}{1} & \multirow{2}{*}{0} & \multirow{2}{*}{804} & 254 & NO \\
\hline & & & & & 282 & NO \\
\hline & \multirow{2}{*}{0} & \multirow{2}{*}{1} & \multirow{2}{*}{1} & \multirow{2}{*}{804} & 253 & YES (with PC 16:1_18:0) \\
\hline & & & & & 282 & NO \\
\hline & \multirow{2}{*}{1} & \multirow{2}{*}{0} & \multirow{2}{*}{1} & \multirow{2}{*}{804} & 254 & No \\
\hline & & & & & 281 & YES (with PC 16:0_18:1) \\
\hline & \multirow{2}{*}{2} & \multirow{2}{*}{0} & \multirow{2}{*}{0} & \multirow{2}{*}{804} & 255 & YES (with PC 16:0_18:1) \\
\hline & & & & & 281 & YES (with PC 16:0_18:1) \\
\hline & \multirow{2}{*}{0} & \multirow{2}{*}{2} & \multirow{2}{*}{0} & \multirow{2}{*}{804} & 253 & YES (with PC 16:1_18:0) \\
\hline & & & & & 283 & YES (with PC 16:1_18:0) \\
\hline & \multirow{2}{*}{0} & \multirow{2}{*}{0} & \multirow{2}{*}{2} & \multirow{2}{*}{804} & 253 & YES (with PC 16:1_18:0) \\
\hline & & & & & 281 & YES (with PC 16:0_18:1) \\
\hline \multirow{14}{*}{ PC 16:0_18:2 } & \multirow{2}{*}{0} & 0 & 0 & 802 & 255 & N/A \\
\hline & & & & out & 279 & N/A \\
\hline & 1 & 1 & 0 & 804 & 256 & NO \\
\hline & & & & & 280 & NO \\
\hline & 0 & 1 & 1 & 804 & 255 & YES (with PC 16:0_18:1) \\
\hline & & & & & 280 & NO \\
\hline & 1 & 0 & 1 & 804 & 256 & NO \\
\hline & & . & $t^{2}$ & r & 279 & NO \\
\hline & 2 & 0 & 0 & 804 & 257 & NO \\
\hline & & . & . & r & 279 & NO \\
\hline & 0 & 2 & 0 & 804 & 255 & YES (with PC 16:0_18:1) \\
\hline & & & & & 281 & YES (with PC 16:0_18:1) \\
\hline & 0 & 0 & 2 & 804 & 255 & YES (with PC 16:0_18:1) \\
\hline & & & & & 279 & NO \\
\hline
\end{tabular}

${ }^{+}{ }^{13} \mathrm{C}$ is used as an example here for simplicity's sake and because it is the most significant contributor to isotopic overlap, but other naturally occurring heavy isotopes are considered in all calculations

Figure 3. Isotopic overlap in MRM transitions using fatty acyls. A) Structures of PC 16:1 18:1, PC 16:0 18:2, PC 16:0 18:1 and PC 16:1_18:0 showing MS/MS fragments and respective MRM transitions; B) Table illustrating in which case(s) isotopic overlap occurs in MRM analysis. 
Figure S4
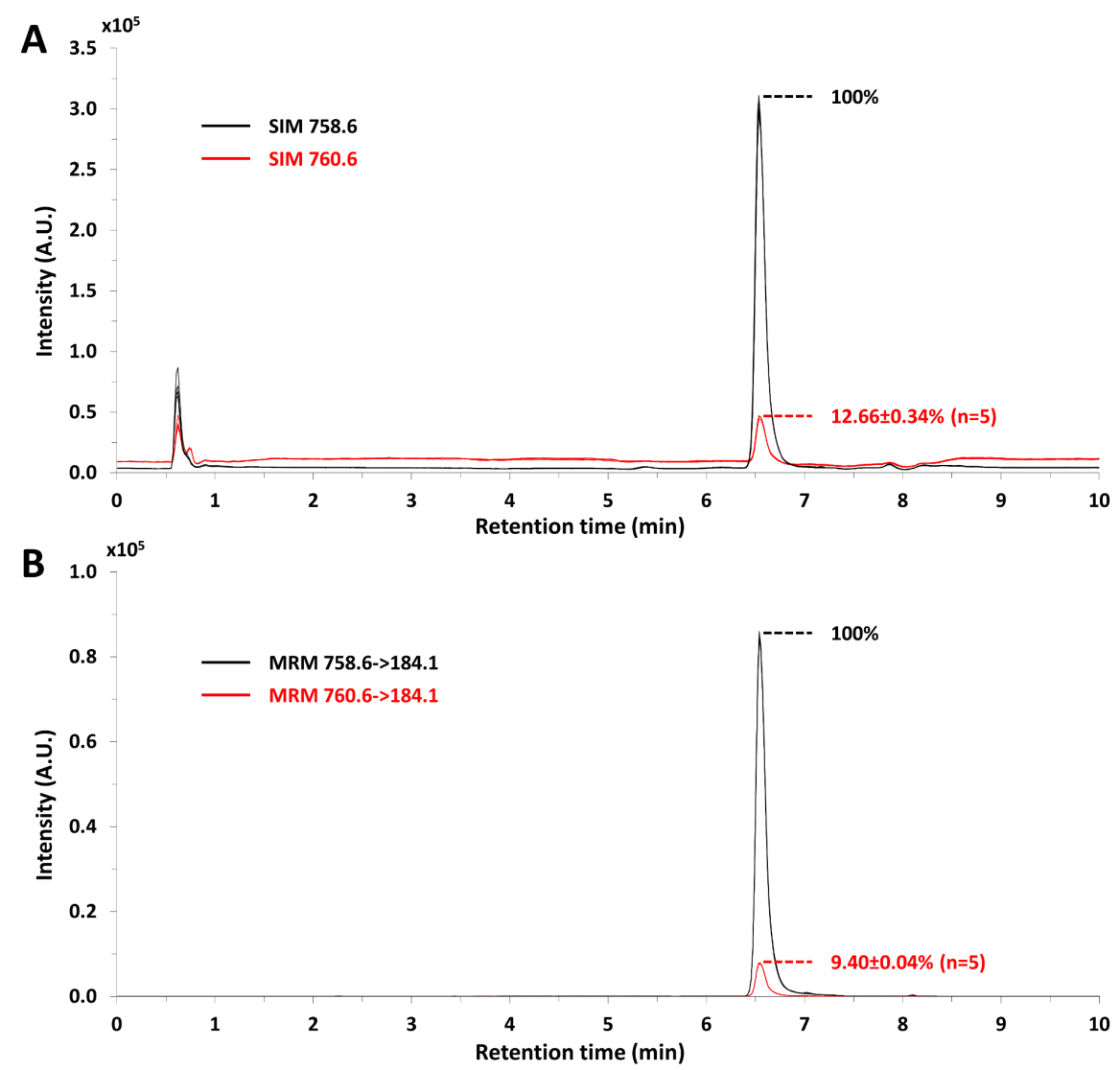

Figure S4. Comparison of the intensity of the isotopic contribution from PC 34:2 as observed in A) SIM analysis and B) MRM analysis. The isotopic contribution is smaller in MRM analysis because the contribution does not come from all atoms but only those in the part of the molecule relevant to the fragment used in the MRM transition. Chromatograms are overlaid from five independent injections. 
Figure S5
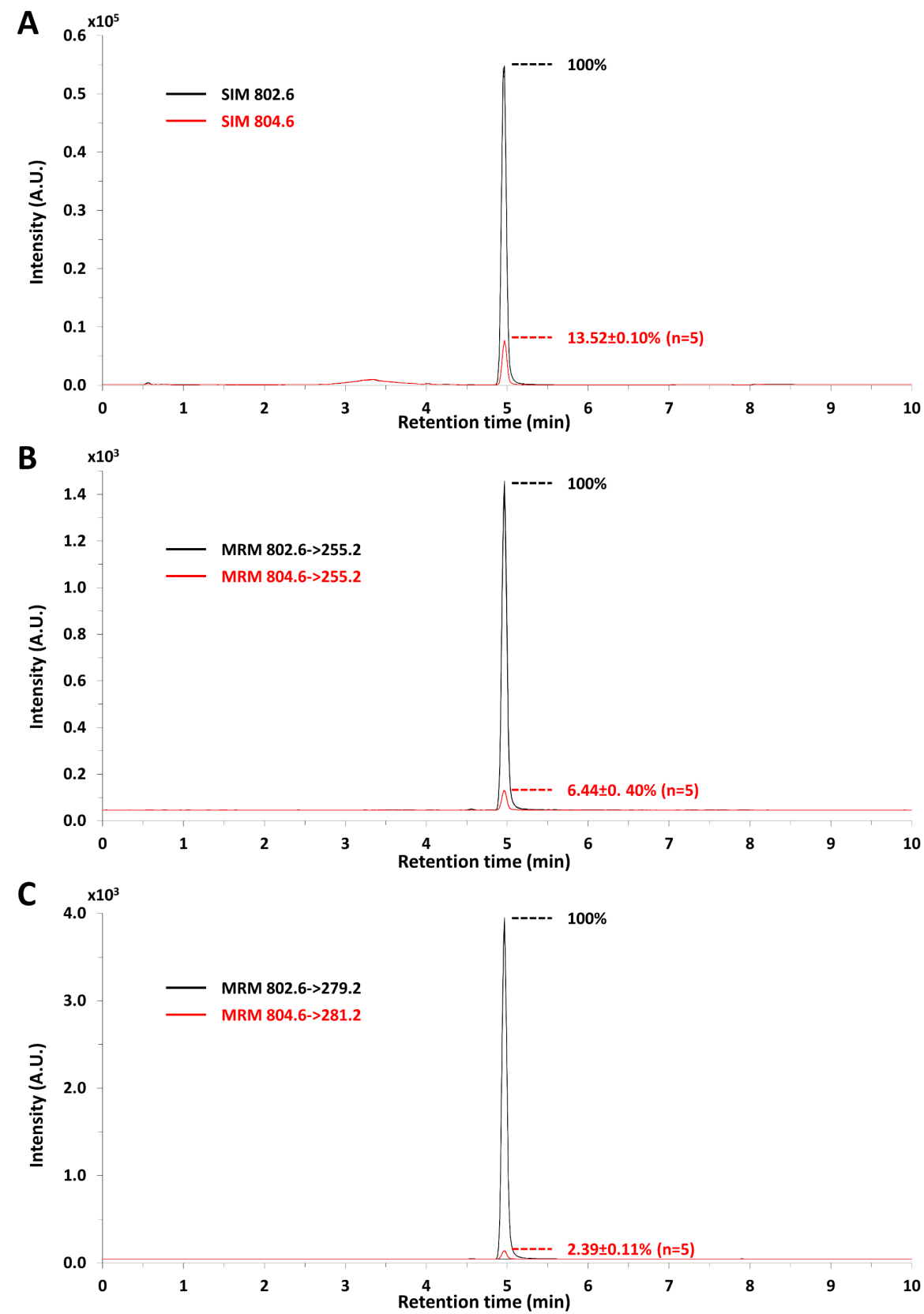

Figure S5. Comparison of the intensity of the isotopic contribution from PC 16:0_18:2, as observed in A) SIM analysis and B) MRM analysis using transition to 16:0 fatty acyl, and C) MRM analysis using transition to 18:2 fatty acyl. The isotopic contribution is smaller in MRM analysis because the contribution does not come from all atoms but only those in the part of the molecule relevant to the fragment used in the MRM transition. Chromatograms are overlaid from five independent injections. 